\title{
Hemoglobin Lansing (Alpha) [HBA2 CD87 (HIS>GLU) $(C>A)]$ in a Turkish Individual Resulting from Another Nucleotide Substitution
}

\author{
Bir Türk Bireyde Başka Bir Nükleotid Substitusyonu \\ Sonucu Gelişen Hemoglobin Lansing (Alpha) [HBA2 CD87 \\ $(H I S>G L U)(C>A)]$
}

Nejat Akarl, Didem Torun², Ayşenur Öztürk²

${ }^{1}$ TOBB-ETU Hospital, Clinic of Pediatrics, Ankara, Turkey

2Ankara University Faculty of Medicine, Department of Pediatric Molecular Genetics, Ankara, Turkey

To the Editor,

Several hemoglobin variants, including novel ones, have been reported in the Turkish population $[1,2,3]$. Herein, we describe a novel nucleotide alteration of the alpha-2 chain variant, hemoglobin (Hb) Lansing CD87 (HIS>GLU).

The index case was a 21-year-old Turkish woman living in Ankara. She was admitted to the TOBB-ETU University Pediatrics Outpatient Department for premarital counseling. Her physical examination was normal. Hemoglobin, hematocrit, and MCV values were $13.1 \mathrm{~g} / \mathrm{dL}$, $42.7 \%$, and $95 \mathrm{fL}$, respectively. Levels of $\mathrm{Hb} \mathrm{A1}, \mathrm{Hb} \mathrm{A} 2$, and $\mathrm{Hb} \mathrm{X}$ were observed as $65.5 \%, 1.88 \%$, and $22.24 \%$, respectively, with high-pressure liquid chromatography. Written informed consent for genetic analysis was obtained from the patient.

DNA was isolated from a peripheral blood sample with the phenol-chloroform protocol. All of the exons of the HBB, HBA1, and HBA2 genes were amplified by polymerase chain reaction (PCR). The entire coding and intronic sequences of the alpha-1 and alpha-2 globin genes were amplified as one amplicon each. While the forward primer was the same for the 2 genes, the reverse primers were specific to the alpha-1 and alpha-2 genes. These amplicons were sequenced using internal primers as described previously [4,5]. PCR products were cleaned with a PCR purification kit (Roche, Germany) and then samples were sequenced using an automatic DNA Sequencer (Beckman Coulter, USA). The DNA was also tested for the - $\alpha 3.7,-\alpha 4.2,-M E D$, and $-\alpha 20.5$ deletions using multiplex PCR according to the described methods $[6,7]$.

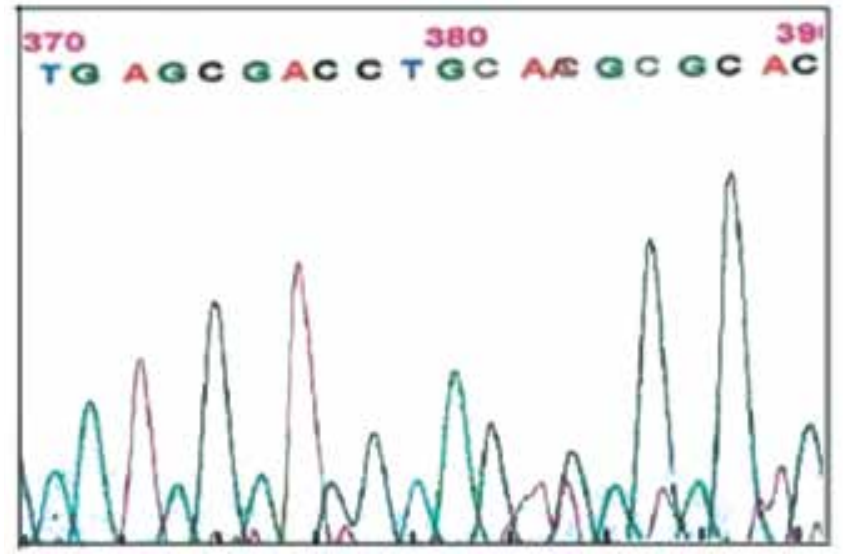

Figure 1. HbA2 gene $264 \mathrm{C}>\mathrm{A}$ transition at exon 2. 848586878889

Address for Correspondence: Didem TORUN, M.D.,

Ankara University Faculty of Medicine, Department of Pediatric Molecular Genetics, Ankara, Turkey

E-mail: didemtorun@gmail.com 
A novel missense mutation was found in codon 87 (CAC>CAA) in the heterozygous state. The sequencing data showed the variant as a mutation at codon 87 of $C$ changing to A, leading to histidine substitution to glutamine, which was not previously described (Figure 1).

There is only one mutation reported at codon 87 of the alpha- 2 gene by Sarikonda et al. They reported a missense mutation at alpha-2 gene codon 87 with substitution of CAC to CAG (His>Gln) and they named this variant Hb Lansing [5]. The reported patient showed the heterozygous form and was asymptomatic with low pulse oximeter readings in the index case. Arterial blood gas showed an $\mathrm{O} 2$ saturation of $98 \%$ on room air [8]. The proportion of the variant in family members was between $10.6 \%$ and $24.2 \%$ [6]. Our patient was also asymptomatic. However, we did not have the chance to further investigate the patient for these above-mentioned clinical symptoms. Other than this, in Asia, Ishitsuka et al. reported a case of $\mathrm{Hb}$ Lansing detected by false low oxygen saturation on pulse oximetry [7].

The variant that we describe is identical in its protein structure to $\mathrm{Hb}$ Lansing. In both cases, the nucleotide change in codon 87 leads to a glutamine residue: CAA in our case and CAG in $\mathrm{Hb}$ Lansing. In human coding regions, CAG is much more common in terms of frequency of usage per thousand (32.95) and relative frequency among synonymous codons (0.73). For CAA, these values are 11.94 and 0.27 , respectively. Therefore, this case is described with codon usage bias [8]. A similar situation was also reported previously in the case of $\mathrm{Hb}$ Niigata. $\mathrm{Hb}$ Nigata is an alteration of valine to leucine at beta-globin gene codon 1 with a nucleotide change of $\mathrm{G}>\mathrm{C} / \mathrm{T}$, which was reported in a Japanese and a Romanian. Moradkhani et al. named the variant of the Romanian individual Hb Niigata (C) $[9,10]$. Located in different genes, the $\mathrm{Hb}$ Niigata and $\mathrm{Hb}$ Lansing variants can be explained by the wobble hypothesis. According to the wobble hypothesis, base pairs are relaxed at the third position, so a base can pair with more than one complementary base. In these variants, changes occur in the third base (CAA>CAG) [11].

As $\mathrm{Hb}$ Lansing was reported previously, we named this new variant $\mathrm{Hb}$ Lansing (A).

Since Turkey is located at the intersection of 3 continents, it is not surprising that many different hemoglobin variants are observed.

\section{Conflict of Interest Statement}

The authors of this paper have no conflicts of interest, including specific financial interests, relationships, and/ or affiliations relevant to the subject matter or materials included.
Key Words: $\mathrm{Hb}$ Lansing, Abnormal hemoglobin, Alpha-globin

Anahtar Sözcükler: Hb Lansing, Anormal hemoglobin, Alfa globin

\section{References}

1. Dungul DC, Ozdag H, Akar N. Hemoglobin alpha 2 gene +861 G>A polymorphism in Turkish population. Egyptian Journal of Medical Human Genetics 2011;12:59-62.

2. Akar E, Ozdemir S, Timur IH, Akar N. First observation of homozygous hemoglobin Hamadan (B 56 (D7) GLY$\mathrm{ARG})$ and beta thalassemia (-29 G>A)-hemoglobin Hamadan combination in a Turkish family. Am J Hematol 2003; 74:280-282.

3. Oron-Karni V, Filon D, Oppenheim A, Rund D. Rapid detection of the common Mediterranean alpha-globin deletions/rearrangements using PCR. Am J Hematol 1998;58:306-310.

4. Tan AS, Quah TC, Low PS, Chong SS. A rapid and reliable 7-deletion multiplex polymerase chain reaction assay for $\alpha$-thalassemia. Blood 2001;98:250-251.

5. Sarikonda KV, Ribeiro RS, Herrick JL, Hoyer JD. Hemoglobin Lansing: a novel hemoglobin variant causing falsely decreased oxygen saturation by pulse oximetry. Am J Hematol 2009;84:541.

6. Pennsylvania State University. Globin Gene Server. http:// globin.bx.psu.edu/cgi-bin/hbvar/.

7. Ishitsuka K, Uchino J, Kato J, Ikuta M, Watanabe K, Matsunaga A, Tamura K. First reported case of hemoglobin Lansing in Asia detected by false low oxygen saturation on pulse oximetry. Int J Hematol 2012;95:731-773.

8. Guigo R. DNA composition, codon usage and exon prediction. In: Bishop MJ (ed). Genetic Databases. San Diego, CA, USA, Academic Press, 1999.

9. Ohba Y, Hattori Y, Sakata S, Yamashiro Y, Okayama N, Hirano T, Nakanishi T, Miyazaki A, Shimizu A. Hb Niigata [ $\beta 1$ (NAl) Val-->Leu]: the fifth variant with retention of the initiator methionine and partial acetylation. Hemoglobin 1997;21:179-186.

10. Moradkhani K, Henthorn J, Riou J, Phelan L, Prehu C, Wajcman H. Hb Niigata [ $\beta 1(\mathrm{NA1}) \mathrm{Val}-->\mathrm{Leu}]$ in a Romanian individual resulting from another nucleotide substitution than that found in the Japanese. Hemoglobin 2007;31:477-482.

11. Crick FHC. Codon-anticodon pairing: the wobble hypothesis. J Mol Biol 1966;19:548-555. 